\title{
Keynote Speech: \\ A Review of the Ontological Status, Computational Foundations and Methodological \\ Processes of Agent-Based Modeling and Simulation Approaches: Open Challenges and Research Perspectives
}

\author{
Alexis Drogoul \\ IRD, UR079-GEODES, 32 av. Henri Varagnat, 93143 Bondy Cedex, France \\ alexis.drogoul@gmail.com
}

Agent based modeling (ABM) and simulation techniques are now used in a number of domains, ranging from social sciences, ecology or biology to exact sciences like physics. They provide modelers with the ability to understand and reproduce, through virtual experiments, the emergence of nearly any kind of macro-structure or macro-dynamics from the interactions of lower level computer programs called agents. As these agents can be programmed with all the details required and can arbitrarily cover any level of representation, ABM undeniably represents one of the most versatile approaches to understanding complex systems through simulation.

However, a lot of criticisms have also been expressed against them, which roughly fall into three categories:

- Criticisms regarding the ontological status of agent-based models in scientific research and that of the results they provide.

- Criticisms (not unrelated to the previous ones) regarding the difficulty of design, calibration, manipulation and validation of these models.

- Criticisms regarding their apparent inability to cope with multiple levels of abstraction at once, in both modeling and simulation.

My talk will try to reply to these criticisms in the light of successful achievements of ABMs, but also show that most of them, although they can concern other modeling and simulation approaches, are effectively sound. This will give me the opportunity to underline which current and future directions of multiagent researches should be mobilized in order to provide innovative answers and therefore contribute to the reinforcement and credibility of ABMs, especially with respect to the last category of criticisms. 